\title{
The Impact Of Complementarity On Power Supply Reliability Of Small Scale Hybrid Energy Systems
}

Jurasz, Jakub; Beluco, Alexandre; Fausto Alfredo Canales Vega.

\section{Abstract}

Small scale hybrid power systems gain popularity around the world as a viable way of reducing power generation environmental impact, reducing energy cost and increasing power supply reliability. Hybrid systems which are based on variable renewable sources usually utilize the effect of resources temporal, and to a smaller extent, spatial complementarity. Although there is already an extensive body of literature investigating the concept of resources complementarity, they rarely addressed the impact of complementarity on the reliability of a given hybrid system. In this paper we simulate the operation of wind and solar hybrid energy system (with and without battery) for evenly distributed 86 locations in Poland over the period 2010-2016 based on 15 min' time step data. We analyze the impact of resources complementarity (on various time scales: $15 \mathrm{~min}$, hourly and monthly) on the system reliability. To remove the capacity factor (resources availability depends on location) on the results, we select the installed capacity in wind and solar sources in such a way that on an annual scale they generate evenly $50 \%$ of the observed demand ( which is assumed to be constant $=1 \mathrm{~kW}$ ). We investigate the impact on complementarity in the system reliability for hybrids with and without energy storage. The second part of the paper deals with the problem of simulating the system reliability (in terms of Loss of Load Parameter) based on multiple linear regression and artificial neural networks. The results indicate that both temporal complementarity (expressed as coefficient of correlation) and storage capacity has non-linear impact on the hybrid system capacity to cover the load. Generated relations between mentioned factors show how complementarity indices may be used to size the solar-wind hybrids. The follow up studies should concentrate on analyzing the operation of hybrids utilizing more than two energy sources and juxtaposing complementarity based reliability assessment with other methods.

\section{Keywords}

Complementarity; Hybrid Energy Source; Photovoltaics; Reliability; Wind Turbine. 